\title{
ERGEBNISSE DER ZWISCHENEVALUIERUNG DER ERSTEN PHASE DER BREITBANDINITIATIVE
}

SUSANNE BÄRENTHALER-SIEBER UND MICHAEL BÖHEIM

\section{KURZZUSAMMENFASSUNG}

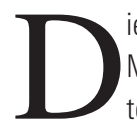
ie Zwischenevaluierung der Breitbandstrategie $2020^{1}$ und des Masterplans ${ }^{2}$ zur Breitbandförderung zeigt, dass die im Masterplan festgelegte und durch die Ausschreibungen verfolgte Umsetzung geeignet ist, das Ziel, Österreich nahezu flächendeckend mit mindestens $100 \mathrm{Mbit} / \mathrm{s}$ schnellem Internet zu versorgen, auch tatsächlich zu erreichen. Auch für den $5 \mathrm{G}$ Ausbau ist die Strategie der schrittweisen Verdichtung von Glasfaserzugangspunkten eine der wichtigsten Maßnahmen zur Steigerung der $5 G$ Readiness. Dessen ungeachtet wird es insbesondere in der 3. Phase notwendig werden, den Förderungsschwerpunkt massiv auf Glasfaser zu legen.

\section{EINLEITUNG}

Im Winter 2016/17 beauftragte das bmvit ein Konsortium aus WIKConsult und WIFO mit der Evaluierung der ersten Phase der Breitbandstrategie 2020 und des Masterplans zur Breitbandförderung durchzuführen. Der vorliegende Artikel $^{3}$ fasst die wichtigsten Ergebnisse dieser Evaluierung (vgl. Neumann et al., 2017) der Phase 2015/20164 der Breitbandinitiative des bmvit zusammen.

\section{DER GUTACHTENAUFTRAG UMFASSTE DIE EVALUIE- RUNG}

1. des Programmdesigns in Bezug auf die definierten Ziele (bisherige Zielerreichung)

2. der Abwicklung der Förderung (um weitere Optimierungspotentiale aufzeigen zu können) und
3. der Abstimmung kommender Ausschreibungen auf den zukünftigen 5G-Ausbau.

Im nächsten Abschnitt wird kurz die verwendete Methode dargestellt. Der danach folgende Überblick über die Ergebnisse ist folgendermaßen aufgebaut. Einleitend wird ein kurzer Überblick über den Zusammenhang von Breitband und der wirtschaftlichen Entwicklung in Österreich sowie über die Breitbandversorgung Österreichs im internationalen Vergleich gegeben. Danach werden die Ergebnisse zu den drei oben genannten Punkten (Programmdesign, Förderungsabwicklung und Abstimmung zukünftiger Ausschreibungen auf 5G), ergänzt um einen Abschnitt zum quantitativen Bild der Förderung, dargestellt. Zuletzt erfolgt ein kurzer Überblick über die Bundesländer-Breitbandstrategien. Das Kapitel schließt mit einem kurzen Fazit und Ausblick.

\section{METHODE}

Die europäischen Breitbandleitlinien ${ }^{5}$ sehen vor, dass bestimme Beihilfenmaßnahmen einer Evaluierung zu unterziehen sind. Diesbezüglich hat Österreich der Europäischen Kommission einen Evaluierungsplan ${ }^{6}$ vorgelegt. Auf Basis dessen hat die Kommission eine Reihe von weiteren Evaluierungsansätzen und Indikatorprüfungen festgelegt ${ }^{7}$. Allerdings können die darin - zumindest auf Ebene der quantitativen Wirkungsanalysen - aufgezählten Evaluierungsfragen weitestgehend erst dann beantwortet werden, wenn die Fördermaßnahmen zu investiv umgesetzten Projekten geführt haben. Da zu Beginn der Erstellung der Evaluierungsstudie (vgl. Neumann et al., 2017) Anfang des Jahres 2017 der Prozess der ersten Calls erst begonnen hatte und voraussichtlich erst zwei bis drei Jahre später abgeschlossen sein wird, konnte die durchgeführte Evaluierung nur eine erste Zwischenevaluierung der Breitbandstrategie Austria 2020 sein. Im Vordergrund dieser Zwischenevaluierung stehen deshalb die Prozesplans. Infolge des (teilweise) verschobenen Starts der Förderprogramme durch die gerichtliche Anfechtung der Ergebnisse der Frequenzauktion hat sich der Abschluss der ersten Phase des Masterplans jedoch in das Jahr 2017 hingezogen, daher konnte die Phase 1 nicht ganz vollständig in die Evaluierung einbezogen werden.

5 Vgl. Europäische Kommission (2013, Randnummer 53).

$6 \quad$ Vgl. bmvit (2014c).

7 Im Detail siehe Neumann et al. (2017, S. 3ff.). 
sanalyse und die Abstimmung der einzelnen Programme untereinander sowie die Effizienz der Abwicklung der Fördermaßnahmen.

Zur Durchführung dieser Zwischenevaluierung wurden zunächst umfassende Fact Finding Missions und Interviewrunden mit den für die Förderung Verantwortlichen des bmvit sowie der Abwicklungsstelle FFG durchgeführt. Um eine Einschätzung der beteiligten Unternehmen und Gebietskörperschaften zum Förderprozess, aber auch zum Programmdesign zu gewinnen, wurden eine Reihe von weiteren Interviews ${ }^{8}$ geführt. Die Erkenntnisse daraus sind in den Evaluierungsbericht gemäß den Einschätzungen und Bewertungen der Studienautoren eingeflossen.

\section{ERGEBNISSE}

\subsection{BREITBAND UND WIRTSCHAFTLICHE ENTWICK- LUNG IN ÖSTERREICH}

Breitbanddatennetze sind ein wichtiger Teil der modernen Infrastruktur, da sie die Grundlage für viele Informations- und Kommunikationsanwendungen und damit das technologische Rückgrat der digitalen Transformation von Wirtschaft und Gesellschaft sind. Die Ergebnisse einer Vielzahl von Studien ${ }^{9}$ zeigen, dass sich die Nutzung von Breitband positiv auf makroökonomischen Dimensionen auswirkt. Beispielsweise hat das WIFO (vgl. Peneder et al., 2016) auf Basis eines Input-Output-Modells herausgearbeitet, dass zusätzliche Investitionen in die Digitalisierung in Höhe von 1 Mrd. € in Österreich Effekte an verbundener Wertschöpfung in Höhe von 1,2 Mrd. € bewirken und dadurch direkt Arbeitsplätze für 14.700 Beschäftigte geschaffen und gesichert werden ${ }^{10}$. Zudem lassen Investitionen in bessere Datennetze positive Wirkungen auf Innovationen erwarten, nicht zuletzt durch den hohen Technologie- bzw. F\&EGehalt der Infrastruktur.

\section{2 ÖSTERREICHS BREITBANDVERSORGUNG IM IN- TERNATIONALEN VERGLEICH}

Österreich weist bereits eine relativ hohe NGA-Abdeckung ${ }^{11}$ auf, diese stützt sich im Wesentlichen auf FTTC/VDSL ${ }^{12}$. Zwar tragen auch die Kabelnetze zur NGA-Abdeckung bei, doch stellt sich die Kabel- und die FTTC/VDSL-Abdeckung als weitgehend überlappend dar. Unterhalb des europäischen Durchschnitts liegt in Österreich die Verfügbarkeit von Breitbandanschlüssen mit mehr als $100 \mathrm{Mbps}$. Die Verfügbarkeit von ultra-schnellen Breitbandzugängen wird im Wesentlichen durch die Kabelnetze dargestellt. Einen noch deutlicheren Aufholbedarf weist Österreich (ebenso wie Deutschland) im Bereich der Glasfasernetze auf.

Auffällig ist in Österreich die - im internationalen Vergleich - relativ geringe Nachfrage nach Anschlüssen mit hoher Geschwindigkeit. Dies gilt sowohl für Bandbreiten größer $30 \mathrm{Mbps}$ als auch für Bandbreiten größer als 100 Mbps. Nur 13\% aller Haushalte fragten 2015 in Österreich einen schnellen Breitbandanschluss (>30 Mbps) nach. In der EU insgesamt waren es zum Vergleich 22\%. Die führenden Länder Belgien und Niederlande wiesen dagegen bereits Anteile von 60\% bzw. 58\% auf ${ }^{13}$. Dies zeigt einen noch wesentlich größeren Abstand zu den europäisch führenden Ländern bei Angebot und Nachfrage nach Hochgeschwindigkeitsanschlüssen.

\subsection{PROGRAMMDESIGN VON BREITBAND AUSTRIA 2020}

Im Rahmen der Breitbandstrategie 2020 werden drei große Infrastrukturprogramme - Access $^{14}$, Backhaul ${ }^{15}$ und Leerverrohrungsprogramm $^{16}$ - umgesetzt, welche durch ein Anwendungs- und ein Anbindungsförderprogramm ergänzt werden. Das Backhaul-Programm auf der einen Seite und das Access- und Leerverrohrungsprogramm auf der anderen Seite sind komplementär zueinander. Insofern sind auf dieser Ebene auch im Großen nahezu keine Synergien und auch keine Konflikte festzustellen. Potentiell kann es nutzbare Synergien zwischen dem Backhaul- und den beiden anderen Programmen geben. Diese können sich ergeben, wenn es in Fördergebieten des Access- und des Leerverrrohrungsprogramms anbindbare Mobilfunkbasisstationen gibt, die (noch) nicht glasfasermäßig angeschlossen sind. Es kann kosteneffizient sein, bei Glasfaserausbauprojekten die Anbindung von Mobilfunkstationen zu realisieren.

Der Förderwettbewerb innerhalb der gleichen Region war in den bisherigen Förderaufrufen sehr schwach ausgeprägt. Im Bereich von Access gab es praktisch keinen Wettbewerb bei FTTC, da nahezu ausdie RTR insbesondere über das Infrastrukturverzeichnis, die Zugangsauflagen und die Wettbewerbswirkungen der Förderung interviewt. Mit dem österreichischen Gemeindeverbund wurden die Anliegen und Bewertungen der Gemeinden zur Förderung erörtert. Darüber hinaus gab es die Gelegenheit erste Ergebnisse der Evaluierung mit den Beauftragten der Bundesländer zur Breitbandentwicklung im Rahmen der Lenkungsausschusssitzung am 29. März 2017 im bmvit zu erörtern. Im Vorfeld hatten die Länder eine Reihe von Informationen zur Breitbandpolitik in ihrem Bundesland in Beantwortung eines Fragebogens, der an sie adressiert wurde, bereitgestellt.

$9 \quad$ Für einen Überblick siehe Friesenbichler (2012) bzw. Neumann et al. (2017).

10 Die sektorale Verteilung der Wertschöpfungseffekte zeigt, dass - aufgrund der hohen Verflechtung - die größten Effekte auf den Bausektor, die Herstellung von Waren sowie den Handel entfallen (vgl. Peneder et al., 2016).

11 Next Generation Access steht für Breitbandzugänge mit einer Downloadgeschwindigkeit von mehr als 30 Mbit pro Sekunde.

12 FTTC steht für "Fibre to the Curb" oder "Fibre to the Cabinet". Dabei handelt es sich um Glasfaserleitungen, welche an einem Straßennetzknoten enden, der sich nahe beim Endkunden befindet. Auf der letzten Wegstrecke werden Kupfer- (bei FTTC/VDSL-Netzen) oder Koaxialkabel (bei Kabel/DOCSIS 3-Netzen) verlegt (vgl. bmvit, 2017). FTTC kann als Zwischenschritt in Richtung Glasfaseranschluss (FTTH oder „Fibre to the Home") gesehen werden.

13 Auswertungen basierend auf Digital Scoreboard 2016 der Europäischen Kommission

14 Das Access-Programm zielt darauf ab, größere Flächen mit leistungsstarken Breitbandzugangsnetzen zu versorgen (vgl. bmvit, 2017), also Investitionen in die Flächenausdehnung zu stimulieren (vgl. bmvit, 2018).

15 Das Backhaul-Programm unterstützt die Verstärkung der Zubringernetze (vgl. bmvit, 2017). Diese Modernisierung bestehender Backhaul-Netze bzw. die Anbindung von Basisstationen soll dazu dienen, bereits bestehende oder zukünftige NGA-Netze mit ausreichender Kapazität zu versorgen (vgl. bmvit, 2018).

16 Das Leerverrohrungsprogramm soll bei geplanten kommunalen Tiefbauarbeiten die Mitverlegung von Leerverrohrungen für Kommunikationsnetze unterstützen (vgl. bmvit, 2017). 
schließlich A1 Telekom diesbezügliche Förderanträge gestellt hat. In einzelnen Regionen gab es Wettbewerb zwischen FTTC und FTTH Projekten. Wettbewerb manifestierte sich hauptsächlich in der Überlappung beantragter Fördergebiete. Im Wesentlichen sind zwei Ursachen für den enttäuschenden Förderwettbewerb feststellbar; einerseits der mangelnde Wettbewerb im Markt generell und andererseits (auch) die konkrete Ausgestaltung des Förderdesigns.

Der Festnetzwettbewerb auf infrastruktureller Ebene ist in Österreich (bislang bis auf wenige Ballungsgebiete) äußerst schwach ausgeprägt. Es gibt bezogen auf ihre aktuelle Marktrelevanz nur wenige ernstzunehmende Wettbewerber zur A1 Telekom. Das ist ein grundsätzlich wettbewerbs- und regulierungspolitisches Problem, dessen Lösung nicht von einem Subventionsprogramm (allein) erwartet werden kann. Auf den Punkt gebracht: Wo kein Wettbewerb herrscht, kann sich auch kein Förderwettbewerb einstellen. Durch die von der Europäischen Kommission ohne Auflagen genehmigte Übernahme von UPC durch T-Mobile entsteht mit Wirksamwerden im Jahr 2019 ein neuer integrierter Telekommunikationsanbieter, sodass pro futuro mit einer Belebung des (potenziellen) Wettbewerbs zu rechnen ist. Der "günstige Wind“ dieser Marktentwicklung wird die österreichische Wettbewerbs- und Regulierungspolitik aber nicht aus der Verantwortung entlassen können, ihre Strategien zu überdenken und die Implementierung von wettbewerbsbelebenden Initiativen substantiell zu verstärken.

Hinzukommt allerdings, dass sich durch die Förderung die relative Wettbewerbsposition des Marktführers A1 Telekom sowohl im Festnetz als auch im Mobilfunk (weiter substantiell) verbessert hat. Soll dieser Effekte nicht weiter verstärkt, sondern abgeschwächt werden - wofür es die bereits genannten wettbewerbs- und regulierungspolitischen Gründe gibt - muss die Förderstrategie mittels eines überarbeiteten Förderdesigns auf diese (unerwünschte) Situation reagieren. Im Mobilfunk sieht die Studie dazu als Ansatzpunkt die deutlich stärkere Förderung von Kooperationsmodellen zwischen Mobilfunkbetreibern und anderen Infrastrukturträgern vor. Beispielsweise könnte der direkte Erwerb von IRUS ${ }^{17}$ direkt förderfähig werden. Im Festnetz-Bereich könnte und sollte die Förderung stärker auf FTTH ausgerichtet werden. Auch die Möglichkeit, dass sich die Förderwerber den Zuschnitt ihres beantragten Fördergebiets als Teil einer NUTS 3-Region selbst festlegen können, sollte deutlich eingeschränkt werden.

Eine kleinräumig definierte Förderkarte wie die österreichische beinhaltet grundsätzlich ein strukturelles Problem. Fördergebiete stellen sich dann oft als nicht zusammenhängende einzelne Kacheln in einem größeren Ausbaugebiet dar. Das Förderregime sollte jedoch nicht einen Netzausbau nach dem "Fleckerlteppichmuster" der Förderkarte herbeiführen, sondern einen ökonomisch effizienten Netzausbau. Größer definierte Fördergebiete beinhalten dagegen das Risiko der „Übersubventionierung", d.h. der Generierung von Mitnahmeeffekten der Förderung. Dieses Problem ist umso größer, je geringer die Intensität des Förderwettbewerbs ist. Der Förderwettbewerb innerhalb der gleichen Region ist in Österreich jedoch eher schwach ausgeprägt. Dies spricht dann aber eher für einen Förderkartenansatz mit kleinen Rastern, um die Förderung auf Gebiete zu beschränken, in denen keine wirtschaftliche Versorgung ohne Förderung darstellbar ist. Die Studie empfiehlt - trotz des erwähnten strukturellen Problems - daher ein Festhalten am bisherigen Förderkartenansatz.

Zum Programmdesign und zu den Bewertungsansätzen der Förderung wurden folgende Reformvorschläge erarbeitet ${ }^{18}$ :

1. Stärkere Berücksichtigung der Vorteile von Glasfasernetzen

2. Anpassung der Mindestgrenzen der Förderung, da zu gering

3. Stärkere Berücksichtigung von Wettbewerbsaspekten bei den Bewertungskriterien

4. Sicherstellung ausreichender Glasfaserkapazität der PoP ${ }^{19}$-Anbindung für die spätere Aufrüstung auf FTTH

5. Stärkere Verzahnung des Anbindungsförderungsprogramms mit dem Access-Programm

6. Anpassung der Kostensätze an aktuelle Marktpreise

7. Erleichterte Möglichkeiten der Infrastrukturübertragung

8. Behandlung des Erwerbs von IRUs an Leerrohren und/oder Glasfaserverbindungen als förderbare Investitionen ohne einschränkende Bedingungen

9. Differenzierte Bewertung von P2P und P2MP-Glasfaser-Topologien $^{20}$

10. Obergrenzen für die Förderung je unversorgtem Wohnsitz

11. Deckungsfähigkeit der Fördermittel zwischen den drei Programmen in der dritten Projektphase

12. Modifizierung der Förderbudgetmittelzuteilung auf Regionen in der zweiten und dritten Programmphase

13. Stärkere Berücksichtigung der Aufrüstung neu versorgter Anschlüsse auf $100 \mathrm{Mbps}$

14. Präferierung von Bewerbern in der 3. Phase, die alle (bislang) unversorgten Wohnsitze in einer NUTS 3-Region versorgen

15. Keine FTTC-Förderung mehr in der dritten Programm-Phase

16. Die Backhaulförderung sollte sich auch auf die Glasfaseranbindung neuer PoPs/Sites beziehen.

\subsection{DAS QUANTITATIVE BILD DER BISHERIGEN FÖR- DERUNGEN (PHASE 1) UND DEREN EFFEKTE}

Für die in 2015/16 initiierten Ausschreibungen Access 1, Backhaul 1 sowie Leerrohr 1 \&2 wurden Fördermittel in Höhe von insgesamt 293 Mio. € zur Verfügung gestellt. Die Mittel des Access 1 Aufrufs waren zweifach überzeichnet und wurden zu $99 \%$ abgeholt. Demgegenüber konnten nur ca. $40 \%$ der Budgetmittel für Leerrohr und $71 \%$ der für Backhaul allozierten Mittel vergeben werden. In Summe wurden in 2015/16 von 293 Mio. € budgetierten Mittel 204 Mio. € vergeben. Die Förderbudgetmittel wurden - seitens der Politik - ex ante auf Bundesländer bzw. NUTS3-Regionen nach einem Schlüssel vergeben, der dem Verhältnis unversorgter zu versorgter Wohnsitze in der entsprechenden kationskapazitäten, die typischerweise mit einer Einmalzahlung abgegolten werden, die wirtschaftlich eine Investition darstellt (vgl. Neumann et al., 2017).

18 Für nähere Details siehe Neumann et al. (2017, S. 78ff.).

19 PoP steht für "Point of Presence” (Verteilknotenpunkt).

20 Unter P2P („,Point-to-Point") versteht man eine Netzwerktopologie, bei der jeder Teilnehmer eine eigene Anschlussleitung bis zur Ortszentrale hat. Während bei einer P2MP („Point-to-Multipoint“) Topologie, jeder Teilnehmer nur bis zu einem zwischengeschalteten passiven Netzknoten eine eigene Anschlussleitung hat. Dort werden diese Leitungen dann zu einer gemeinsam genutzten Leitung zusammengefasst (bmvit, 2017). 
Region entsprach. Insgesamt gab es vor dem Start der Förderprogramme etwa 1,9 Mio. (NGA) förderungsfähige unversorgte Haupt- und Nebenwohnsitze; dies entspricht etwa 20\% aller Haupt- und Nebenwohnsitze in Österreich. Nach Implementierung von Phase 1 können durch die Förderungen insgesamt ca. 30\% der bisher unversorgten Wohnsitze nun neuversorgt werden, wenn die geförderten Projekte investiv umgesetzt sind. Am höchsten war dieser Versorgungslückenschluss auf Bundesländerebene in Tirol (fast 50\%), Vorarlberg (ca. 44\%) und Kärnten (42\%). Gefolgt von den Bundesländern Niederösterreich (32\%), Steiermark (24\%) und Burgenland (23\%) sowie Oberösterreich (20\%). Hinsichtlich Neuversorgung bleiben nur Wien (ca. 7\%) und Salzburg (ca. 10\%) deutlich unter dem Österreich Durchschnitt.
Sektor sind diese initialisierten Investitionen erheblich. Im Durchschnitt der letzten Jahre haben die Betreiber p.a. ca. 600 Mio. € (ohne Frequenzaufwendungen) in die Netzinfrastruktur investiert. Durch die Förderung kann dadurch das im internationalen Vergleich eher geringe Niveau der TK-Investitionen in Österreich (deutlich) angehoben werden.

Die Investition pro neu versorgtem Wohnsitz in Österreich betrug im Durchschnitt $488 €$. Im Access 1 Programm wurden rund $400 €$ pro neu versorgtem Wohnsitz (im österreichischen Durchschnitt) investiert. Für die Leerrohrprogramme 1 und 2 fielen durchschnittliche Investitionskosten von rund $1.000 €$ pro neu versorgten Wohnsitz an. Im Durchschnitt war $\mathrm{FTTH}^{21}$ im Ausbau der Phase 1 die teuerste Technologie (1.654 €) gefolgt von $\mathrm{FTTB}^{22}$ (1.112€), deutlich günstiger kamen $\mathrm{FTTC}^{23}$ (xDSL) (475 $€)$ und Mobilfunk (252€).

\begin{tabular}{|c|c|c|c|c|c|c|c|c|c|c|c|}
\hline Bundesländer & Unv. WS & Vers. WS & $\begin{array}{c}\text { WS } \\
\text { gesamt }\end{array}$ & $\begin{array}{c}\text { Anteil unv. } \\
\text { WS }\end{array}$ & $\begin{array}{c}\text { neu vers. } \\
\text { WS }\end{array}$ & $\begin{array}{l}\text { Anteil neu } \\
\text { vers. WS } \\
\text { an allen } \\
\text { WS }\end{array}$ & $\begin{array}{c}\text { Anteil neu } \\
\text { vers. WS } \\
\text { an unvers. } \\
\text { WS }\end{array}$ & $\begin{array}{c}\text { Investition } \\
\text { pro neu } \\
\text { vers. WS }\end{array}$ & $\begin{array}{c}\text { Förderung } \\
\text { pro neu } \\
\text { vers. WS }\end{array}$ & $\begin{array}{c}\text { Index } \\
(\hat{0}=100)\end{array}$ & Förderquote \\
\hline Burgenland & 41.700 & 296.400 & 338.100 & $12,3 \%$ & 9.700 & $2,87 \%$ & $23,26 \%$ & $€ 471$ & $€ 235$ & 97 & $50,0 \%$ \\
\hline Niederösterreich & 475.900 & 1.459 .900 & 1.935 .800 & $24,6 \%$ & 152.100 & $7,86 \%$ & $31,96 \%$ & $€ 559$ & $€ 278$ & 115 & $49,8 \%$ \\
\hline Wien & 99.600 & 1.928 .000 & 2.027 .600 & $4,9 \%$ & 7.200 & $0,36 \%$ & $7,23 \%$ & $€ 144$ & $€ 72$ & 30 & $50,0 \%$ \\
\hline Kärnten & 216.300 & 410.400 & 626.600 & $34,5 \%$ & 91.200 & $14,55 \%$ & $42,16 \%$ & $€ 322$ & $€ 161$ & 66 & $50,0 \%$ \\
\hline Steiermark & 457.200 & 884.500 & 1.341 .700 & $34,1 \%$ & 108.800 & $8,11 \%$ & $23,80 \%$ & $€ 330$ & $€ 165$ & 68 & $50,0 \%$ \\
\hline Oberösterreich & 318.700 & 1.251 .100 & 1.569 .800 & $20,3 \%$ & 64.500 & $4,11 \%$ & $20,24 \%$ & $€ 828$ & $€ 409$ & 169 & $49,4 \%$ \\
\hline Salzburg & 32.800 & 581.500 & 614.300 & $5,3 \%$ & 3.400 & $0,55 \%$ & $10,37 \%$ & $€ 1.072$ & $€ 536$ & 221 & $50,0 \%$ \\
\hline Tirol & 198.400 & 628.100 & 826.500 & $24,0 \%$ & 98.300 & $11,89 \%$ & $49,55 \%$ & $€ 544$ & $€ 270$ & 111 & $49,7 \%$ \\
\hline Vorarlberg & 50.000 & 352.400 & 402.400 & $12,4 \%$ & 21.900 & $5,44 \%$ & $43,80 \%$ & $€ 247$ & $€ 123$ & 51 & $50,0 \%$ \\
\hline Österreich gesamt & 1.890 .600 & 7.792 .300 & 9.682 .800 & $19,5 \%$ & 557.100 & $5,75 \%$ & $29,47 \%$ & $€ 488$ & $€ 243$ & & $49,7 \%$ \\
\hline Mittelwerte & & & & & & & & $€ 502$ & $€ 250$ & & \\
\hline
\end{tabular}

Tabelle 1: Breitbandabdeckung je Bundesland ex ante und ex post Phase 1

Quelle: FFG-Daten, WIK/WIFO-Berechnungen, gekürzte Fassung der Tabelle 4-1 aus Neumann et al. (2017) - Daten über versorgte Wohnsitze sind nur für die Programme Access 1 und Leerrohr 1 \&2 verfügbar. - Phase1 war zu dem Zeitpunkt der Auswertung diese Daten noch nicht ganz abgeschlossen, siehe dazu auch Fußnote zur Phase 1 in der Einleitung.

Von den ca. 204 Mio. € an bewilligten Förderungen entfällt fast die Hälfte auf Access 1, etwas mehr als ein Drittel auf Backhaul 1 und das restliche Fünftel auf Leerrohr 1\&2. Bei einer durchschnittlichen Förderintensität knapp am Maximum von $50 \%$ beliefen sich die geförderten Projektkosten auf ungefähr 410 Mio. €. Berücksichtigt man effektiv höhere Projektkosten und die Erforderlichkeit von Investitionen in aktive Netzkomponenten, die nicht gefördert werden, aber von den Fördernehmern beigestellt werden müssen, um die geförderte passive Infrastruktur für Telekommunikations-Zwecke nutzen zu können, ergibt die WIK-Schätzung 503 Mio. € Investitionen, die durch die Förderung initialisiert werden. Der Multiplikator von Förderung zu getätigten Investitionen beträgt demnach 2,5. Das ist deutlich mehr als die vorgesehene Verdoppelung der Förderung durch die privaten Telekombetreiber. Dabei nicht berücksichtigt sind indirekte Effekte durch induzierte Investitionen in Gebieten, die an Fördergebiete angrenzen.

Bezogen auf die früher üblichen Investitionen im österreichischen TK-

\subsection{ABWICKLUNG DER FÖRDERMASSNAHMEN}

Die bisherige Zeitdauer des Förderprozesses macht ein Überdenken des zeitlichen Ablaufs erforderlich. Die Gesamtdauer des ersten LeerrohrCalls erforderte 19 Monate und die des ersten Access-Calls 13 Monate. Die Evaluierungsstudie hält eine Gesamtdauer von 10 Monaten von der Eröffnung eines Förderaufrufs bis zu den Vertragsabschlüssen für angemessen. Wesentlichste Ursache für die lange Dauer der ersten Phase waren Verhandlungen über die Bedingungen der Zugangsgewährung. Im Streitfall muss die Abwicklungsstelle hier zeitgerecht abschließend die Bedingungen festlegen. Gegeben die Komplexität der Breitbandförderung wird der Verwaltungsaufwand auf Seiten der FFG und des bmvit für die Breitbandförderung für angemessen und eher knapp als großzügig bemessen gehalten.

Die Evaluierungsstudie kommt zu den folgenden Reformvorschlägen zur Verbesserung der Abwicklungseffizienz ${ }^{24}$ : 
1. Verkürzung der Zeitdauer des Förderabwicklungsprozesses

2. Zeitliche Entzerrung der Calls für die einzelnen Programme

3. Offener Call für das Leerrohrprogramm

4. Überausschöpfung der Budgetobergrenzen

5. Frühzeitige Einstellung geförderter Projekte in das Infrastrukturverzeichnis.

\subsection{BREITBANDFÖRDERUNG UND BREITBANDSTRA- TEGIE, GUTE VORAUSSETZUNGEN FÜR 5G}

Jede Breitbandstrategie, die zu einem bestimmten Zeitpunkt formuliert wurde, muss sich nach einigen Jahren fragen, ob die Prämissen, die Technologie und den Markt betreffend, auf denen sie aufgesetzt hat, zum aktuellen Zeitpunkt und für die absehbare Zukunft Änderungen erfahren haben bzw. im Planungshorizont erfahren werden. Ähnliches gilt für politische, insbesondere europapolitische Randbedingungen. Die Zwischenevaluierungsstudie sah zum Erstellungszeitpunkt vor allem vier Entwicklungen, die gegenüber den Entscheidungen zur aktuellen Breitbandstrategie inzwischen wichtige Änderungen bzw. Konkretisierungen erfahren haben:

1. Die 5G-Entwicklung ist konkreter und umsetzungsnäher geworden.

2. Die Orientierung auf flächendeckende Glasfasernetze als universelle Festnetzinfrastruktur wird immer klarer und in mehr und mehr Ländern Realität.

3. Die EU ist dabei, die Breitbandziele ihrer Digitalen Agenda neu zu formulieren.

4. Die Nachfrageentwicklung bestätigt den Bedarf nach Bandbreiten deutlich jenseits des 100 Mbps Ziels bereits ab 2025.

Die Erwartungen für $5 \mathrm{G}$ sind sehr optimistisch und suggerieren eine signifikante Bedeutung von $5 \mathrm{G}$ mit makroökonomischer Dimension, vor allem vor dem Hintergrund der digitalen Transformation von Wirtschaft und Gesellschaft (vgl. Bock-Schappelwein \& Böheim, 2018). Insgesamt wird $5 \mathrm{G}$ zu einer Vielzahl disruptiver Anwendungen führen, die in vielen Sektoren wesentliche Innovationen und Produktivitätssteigerungen ermöglichen. Allerdings gibt es auch warnende Stimmen, die darauf aufmerksam machen, dass die Gesamtentwicklung von $5 \mathrm{G}$ nicht überschätzt und vor allem keine zu schnelle zeitliche Realisierung erwartet werden darf. So erwarten viele nicht einen disruptiven, sondern einen evolutionären Übergang auf die 5G-Technologie. Vielfach wird auch in Zweifel gezogen, dass $5 \mathrm{G}$ ein relevantes zusätzliches Umsatzpotential für die Betreiber generieren kann. Hingegen sind erhebliche Investitionen erforderlich, die das Modell des Infrastrukturwettbewerbs in Frage stellen können.

Kein Land kann sich dem technologischen Fortschritt und dem Generationswechsel der Mobilfunktechnologien entziehen. Die technologischen Potentiale von $5 \mathrm{G}$ sind auch in unserer Einschätzung erheblich. Weniger deutlich scheint uns dies aber für die wirtschaftlichen Perspektiven gezeigt zu sein. Ein beschleunigter Technologiewechsel von $3 G / 4 G$ auf $5 \mathrm{G}$ ließe sich wirtschaftlich nur rechtfertigen, wenn die neue Technologiegeneration dies durch zusätzliche Erlöse unterlegt.
Anderenfalls bringt ein beschleunigter Technologiewechsel hohe wirtschaftliche Risiken bis hin zur Existenzgefährdung für die $\mathrm{MNOs}^{25}$. Mit besonders hohen Risiken für die Betreiber scheint daher eine Vorreiterstrategie verbunden zu sein, wie sie etwa ADL (2017) im Auftrag der Internetoffensive propagiert. Die Studie spricht sich allerdings auch nicht für eine Strategie des Abwartens bei $5 \mathrm{G}$ aus. Vielmehr wird eine Strategie der forcierten Verbesserung und Steigerung der "5GReadiness" sowohl gesamtwirtschaftlich für Österreich als auch einzelwirtschaftlich für die Mobilfunkbetreiber als besonders vorteilhaft und effizient gesehen. Sie forciert durch eine Vielzahl von Maßnahmen die 5G-Readiness, baut Hemmnisse ab und schafft gute Voraussetzungen für eine später forcierte Einführung. Die Studie führt in ihrer Bewertung die folgenden Maßnahmen an, mit welchen Österreich seine 5G-Readiness weiter vorantreiben kann.

1. Weitere Steigerung der Netzabdeckung und Netzverdichtung bei $4 \mathrm{G}$.

2. Glasfaseranbindung einer möglichst hohen Anzahl an Mobilfunkstandorten.

3. Flächenausbau eines Glasfasernetzes.

4. Entwicklung eines regulatorischen/wettbewerbsrechtlichen Rahmens, der eine stärkere Netzkooperation der Mobilfunkbetreiber erlaubt.

5. Förderung von Use Cases in Pilotregionen.

6. Senkung der Standortkosten für neue (und ggf. auch bestehende) Sites.

7. Intensivierung der 4G-Nutzung.

8. Förderung von innovativen Anwendungen.

9. Frühzeitige Verfügbarkeit von Frequenzen und Transparenz des Vergabeprozesses.

10. Prozesserleichterung bei der Erschließung und Nutzung neuer Standorte.

11. Kostenfreie Errichtung von Infrastruktur auf öffentlichem und privatem Grund.

12. Leitungsrechte gem. TKG nicht nur für Kabelleitungen, sondern auch für Sendestandorte.

13. Überprüfung und Senkung von Stromanschlusskosten.

14. Entwicklung von großflächigen Pilotanwendungen im Bereich öffentlicher und staatlicher Institutionen.

Die österreichische Breitbandstrategie ist bislang darauf angelegt, dass sich die Infrastrukturentwicklung (primär) evolutionär von NGAKonnektivität zu ultraschnellem Breitband bis zur Gigabitkonnektivität einstellt. Dieser Ansatz hat den strategischen Vorteil, relativ schnell und Förderbudget schonend Flächendeckung mit NGA-Breitband und vielleicht sogar mit ultraschnellem Breitband zu erreichen. Einer Breitbandstrategie, die sich nicht dieser betriebswirtschaftlichen Logik des evolutionären Ausbaus aussetzen und den langsamen Übergang auf Gigabitkonnektivität nicht akzeptieren will, bleibt nur die Option, Gigabitnetze im Rahmen eines Überbaus zu forcieren. Eine derartige Strategie des disruptiven Übergangs kann aus zwei Elementen bestehen:

1. Keine Förderung (mehr) für den FTTC-Ausbau, sondern nur noch für den Leerrohrausbau und von FTTB/H-Access. 
2. Förderung von Leerrohrausbau und FTTH auch in Gebieten, in denen FTTC ausgebaut wurde.

Der Masterplan zur aktuellen Breitbandstrategie muss sich auch bereits für die Umsetzung in der zweiten und dritten Phase stärker auf die weiterentwickelten Ziele ausrichten. Neumann et al. (2017) bieten in ihrer Bewertung der Breitbandinitiative dazu folgende Elemente an:

1. Stärkere Differenzierung der Bewertungskriterien mit Blick auf das Leistungspotential von FTTH;

2. Stärkere Berücksichtigung von $\mathrm{LTE}$, insbesondere aber $5 \mathrm{G}$ bei der Access-Förderung;

3. Stärkere Berücksichtigung des Netzausbaus in ausgedehnteren Ausbaugebieten.

\subsection{BREITBANDSTRATEGIEN DER BUNDESLÄNDER UND DES BUNDES}

Die Bundesländer haben eigene Breitbandstrategien, zum Teil auch eigene umfangreiche Breitbandstrategiedokumente entwickelt. Betreffend die Ziele der Bundesländer-Breitbandstrategien gibt es unterschiedlich ambitioniert gesetzte Zielsetzungen, den Zeitraum insbesondere aber auch die Bandbreite betreffend ${ }^{26}$. Auch die Art der Umsetzung ist unterschiedlich zwischen den Bundesländern. Wie die Analyse gezeigt hat, präsentieren sich die Breitbandstrategien der Bundesländer als sehr individuell und divers (Stichwort: "Jeder geht seinen eigenen Weg"). Idealerweise sollten jedoch die Strategien, Ziele, und Maßnahmen der Bundesländer im Einklang mit den Breitbandstrategien und -zielen des Bundes stehen, diese jedenfalls nicht konterkarieren. Im Wesentlichen nützen die Bundesländer die Breitbandförderung des Bundes, um ihre länderspezifischen Breitbandstrategien umzusetzen und sich diese Umsetzung durch Bundesmittel (ko-)finanzieren zu lassen. Zielorientierte Koordinierungsbemühungen auf beiden Seiten könnten daher noch mehr intensiviert werden. Insbesondere die Abstimmung zwischen den Bundesländern untereinander und mit der Strategie des Bundes ist nicht immer ausreichend. Der Lenkungsausschuss ist ein Gremium, dem hier eine stärkere Rolle zuwachsen könnte ${ }^{27}$. Eine verbesserte Koordination könnte die Effizienz und die Effektivität aller Förderungen, sowohl auf Bundes- als auch auf Länderebene nachhaltig stärken.

\section{FAZIT}

Das bmvit hat sich in der Zwischenzeit auf seiner Website bereits dazu bekannt, die meisten Empfehlungen der Zwischenevaluierungsstudie (insbesondere die Verkürzung der Dauer der Förderabwicklung, mobile Beratung vor Ort sowie verstärkter Fokus auf den Glasfaserausbau) in die Breitbandstrategie des Infrastrukturministeriums einzuarbeiten ${ }^{28}$.

Als Ausblick sei jedoch darauf hingewiesen, dass zumindest in einem Punkt noch Verbesserungsbedarf auch hinsichtlich zukünftiger Evaluationen, nämlich bei der zur Verfügungstellung von Informationen über den Fördermitteleinsatz der Bundesländer, besteht. Da noch keine ein- heitlichen Reportingstandards implementiert sind, wird - aus Sicht der Autoren - eine umfassende Evaluierung unnötig erschwert. Ziel muss es sein, pro futuro alle Subventionen des Bundes und der Länder in einer öffentlich zugänglichen Datenbank zur Verfügung zu haben. Dies ist im Sinne einer evidenzbasierten Wirtschaftspolitik nicht nur für zukünftige quantitative Evaluierungen unabdingbar, sondern auch für die begleitende Projektsteuerung der Förderstellen von großem Nutzen. Diese Informationen fehlen auch für eine bessere Abstimmung der Länderstrategien mit der Breitbandstrategie des Bundes sowie der Breitbandstrategien der Bundesländer untereinander.

\section{LITERATUR}

ADL (2017): Österreich als 5G Vorreiter, Der Weg an die Spitze, Studie im Auftrag der Internet-Offensive Österreich, Januar 2017, Arthur D. Little.

bmvit (2014a): Breitbandstrategie 2020, 2. Auflage, Wien, https://www. bmvit.gv.at/service/publikationen/telekommunikation/downloads/breitbandstrategie2020_ua.pdf (Zugriff: 3. September 2018).

bmvit (2014b): Die ganze Bandbreite des Lebens. Ein Masterplan zur Breitbandförderung, 2. Auflage, Wien, https://www.bmvit.gv.at/service/ publikationen/telekommunikation/downloads/breitband_offensive_ ua.pdf (Zugriff: 3. September 2018).

bmvit (2014c): Provisional supplementary information sheet for the notification of an evaluation plan, version July 2014.

bmvit (2017): Breitband in Österreich, Evaluierungsbericht 2016, Breitbandbüro, Stabstelle Informations- und Kommunikationsinfrastruktur, Wien, 03/2017, https://www.bmvit.gv.at/service/publikationen/telekommunikation/downloads/evaluierung2016.pdf (Zugriff: 3. September 2018).

bmvit (2018): Breitbandförderung: Breitband Austria 2020, https://www. bmvit.gv.at/telekommunikation/breitband/foerderungen/index.html (Zugriff: 3. September 2018).

Bock-Schappelwein, J., Böheim, M. (Koordination) (2018): Politischer Handlungsspielraum zur optimalen Nutzung der Vorteile der Digitalisierung für Wirtschaftswachstum, Beschäftigung und Wohlstand, WIFOStudie im Auftrag des Bundeskanzleramts und des Bundesministeriums für Digitalisierung und Wirtschaftsstandort, Wien (im Erscheinen).

Europäische Kommission (2013): Mitteilung der Kommission, Leitlinien der EU für die Anwendung der Vorschriften über staatliche Beihilfen im Zusammenhang mit dem schnellen Breitbandausbau, (2013/C 25/01), 26.1.2013.

Friesenbichler, K. S. (2012): Wirtschaftspolitische Aspekte des Glasfaserausbaus in Österreich, WIFO, Wien, http://www.wifo.ac.at/wwa/ pubid/44135 (Zugriff: 3. September 2018). 
Neumann, K.-H., Plückebaum, T. (WIK Consult), Böheim, M., Bärenthaler-Sieber, S. (WIFO) (2017): Evaluierung der Breitbandinitiative bmvit - 2015/2016, Auftraggeber: Bundesministerium für Verkehr, Innovation und Technologie", Mai 2017, https://www.bmvit.gv.at/service/ publikationen/telekommunikation/downloads/evaluierung_initiative2017.pdf (Zugriff: 3. September 2018).

Peneder, M., Bock-Schappelwein, J., Firgo, M., Fritz, 0., Streicher, G. (2016): Österreich im Wandel der Digitalisierung, WIF0, Wien, http:// www.wifo.ac.at/wwa/pubid/58979 (Zugriff: 3. September 2018)

\section{AUTORINNEN}

\section{SUSANNE BÄRENTHALER-SIEBER}

WIFO, Arsenal, Objekt 20

1030 Wien

E: Susanne.Baerenthaler-Sieber@wifo.ac.at

\section{MICHAEL BÖHEIM}

WIFO, Arsenal, Objekt 20

1030 Wien

E: Michael.Boeheim@wifo.ac.at 